\title{
DATA ASSIMILATION AND PREDICTABILITY STUDIES FOR THE COUPlED OCEAN-ATMOSPHERE SySTEM
}

\author{
By Michael Ghil and Carlos R. Mechoso
}

\begin{abstract}
As As OCEANIC DATA SETS increase dramatically in quality and quantity in the near future, and both oceanic and atmospheric models improve apace, the predictability of the coupled ocean-atmosphere system will become more important on the theoretical level and more critical on the practical level. Predictability of the atmosphere with prescribed sea-surface temperatures (SST) has been evaluated; numerous studies indicate that two initially very similar atmospheric states will lead to time evolutions that on the average diverge and become uncorrelated over an interval on the order of 2 weeks. There is also a growing literature on the predictability of the upper ocean with prescribed atmospheric wind stress and heat fluxes. But the variability, and hence predictability, of the coupled system is quite different from the sum, product. or any other simple function of its parts (Ghil et al., 1991a).

The long-term goal of our work at UCLA is to provide a description, understanding, and prediction of the coupled ocean-atmosphere system as complete and reliable as that which now exists for the atmosphere alone. Our approach is to develop methods for data assimilation from sequential estimation and control theory and for predictability studies from dynamical systems and statistical turbulence theory; these methods are then tested on a variety of models, ranging from simple models amenable to analytical treatment to coupled ocean-atmosphere general circulation models (GCMs).
\end{abstract}

\section{Data Assimilation For The Coupled Ocean- Atmosphere System}

The ambitious and elusive goal of data assimilation is to provide a dynamically consistent "motion picture" of the atmosphere and oceans

M. Ghil. Department of Atmospheric Sciences and Institute of Geophysics and Planetary Physics, University of California, Los Angeles, CA 90024-1565, USA. C. R. Mechoso. Department of Atmospheric Sciences, University of California. Los Angeles. 90024-1565. USA in three space dimensions with known error bars. The ingredients for generating this four-dimensional space-time movie are a large number of observations with different spatiotemporal distributions and error characteristics, on the one hand, and an imperfect knowledge of and ability to solve the equations of fluid motion, on the other.

The purposes of generating such a movie can differ: in numerical weather prediction and in the emerging discipline of ocean forecasting, the main emphasis is on short "loops" between successive initial states for subsequent prediction, separated by 1 day (in the atmosphere) or 1 week to 1 month (in the oceans). In climate-related problems, whether atmospheric or oceanic, the emphasis is on full-length "feature movies," based on all the information available for long-time intervals, e.g., for the entire duration of a field experiment or of even longer historic data records.

In meteorology, data assimilation is a well-established subfield described in books such as Bengtsson et al. (1981) and Daley (1991). In oceanography, the increase in data sets as well as the improvement in models are working a true revolution in the need for and interest in dataassimilation methods. The general problem of data assimilation for the atmosphere and oceans is discussed by Ghil $(1989,1990)$ and by Ghil and Malanotte-Rizzoli (1991).

A considerable number of methods with increasing degrees of sophistication have been developed for and applied to the assimilation of atmospheric and oceanic data. The key difficulty resides in ascertaining the relative confidence one has in, and therefore the relative weights one should assign to, various observations and various model predictions. In direct insertion, local observations are given complete credence and are used to simply replace model predictions at the time of observation. In variational methods with strong constraints, the model is considered perfect and observations are only allowed to help pick the succession of model predictions that are closest
The. . . goal of data

assimilation is to

provide a dynamically

consistent "motion

picture" of the

atmosphere and

oceans. . . 
to the observations. Other methods try to use different theoretical ideas and computational resources, the common purpose being to assign relative weights to model values and observed values in inverse proportion to their estimated square errors; these methods include successive corrections, nudging, so-called optimal interpolation (OI) and variational methods with weak constraints (Daley, 1991; Ghil and Malanotte-Rizzoli, 1991).

The best estimate of the current state of a geophysical flow field, based on past and current observations, is provided in the case of linear flow equations by the Kalman (1960) filter. The filter makes optimal use of information about the observational errors and the model errors to calculate the above-mentioned relative weights in computing the current flow-field estimate. It can be generalized to the more realistic nonlinear equations used nowadays in dynamic meteorology and physical oceanography via the so-called extended Kalman filter (EKF: Ghil et al., 1981; Miller and Ghil, 1990). At UCLA, we have applied a full suite of the data-assimilation methods sketched above to models of varying complexity and with varying resolutions of the mid-latitude and tropical ocean.

Todling and Ghil (1991) investigated the ability of a data-assimilation system based on the Kalman filter to track rapidly developing barotropic and baroclinic instabilities in the atmosphere and oceans. The simplest way to test the performance of a data-assimilation method, used in conjunction with a given model and a given set of observations, is to run a so-called identical-twin experiment. First, a control integration is carried out with a given initial state; this integration is considered to provide the correct succession of states of the flow fields and therefore is also often named "the nature run." Next, random perturbations are added to the initial state (to reflect our lack of complete and accurate knowledge thereof) and to the model equations; this run is considered to represent the succession of states that we would obtain in the absence of any observations. Finally, an assimilation run is made with the same initial state as in the second, perturbed run, but using observations extracted from the control run at selected locations (according to an existing or planned observing pattern) and is blended with the model-predicted values while applying the weights provided by the assimilation method under study. The difference between the model state at any given time in the assimilation run and that in the control run is referred to as model anomaly and is measured typically by the corresponding root-mean-square (RMS) error; the performance of the data-assimilation method is reflected by the reduction of this error over time with respect to its initial value, or with respect to another appropriate comparison value (e.g., that in the second run, without observations).
For the barotropic case, Todling and Ghil (1991) produced an identical-twin simulation by adding random perturbations to the results of a run performed using a shallow-water model, initialized with an unstable velocity profile (Fig. 1). In the assimilation runs, the observations were assimilated using the Kalman filter method. In this way, Todling and Ghil (1991) showed that the Kalman filter results in a substantial reduction of the initial error, even for a limited number of observations (Fig. 2). Furthermore, they showed that observations made within the region of most unstable flow are more effective for the assimilation. This work is being extended to the baroclinic case; preliminary results for this case were reported by Todling and Ghil (1990).

An important problem for ocean prediction is the compensation of errors in atmospheric windstress data by the use of ocean data. This problem was explored by Hao (1991), using a linear, reduced-gravity model of a tropical ocean basin and an assimilation method based on OI. Hao forced the model with biased wind stresses and compared the effect of assimilating (at selected locations) either the height of the free surface (as would be the case if altimeter data were used) or the zonal velocity (as would be the case if current-meter data were used). Hao found that error reduction due to assimilation varies between the western, central, and eastern parts of the basin, and between the height and velocity field (Fig. 3). The dependence

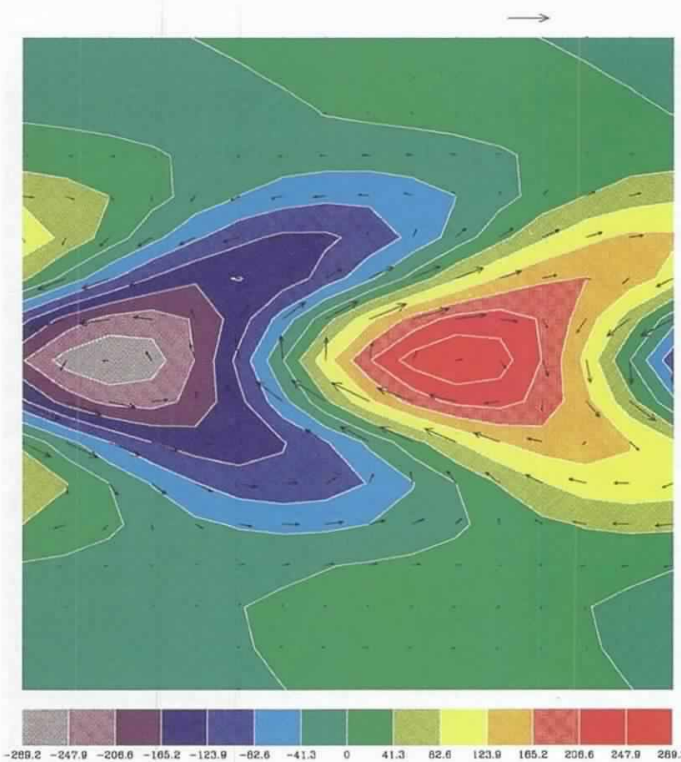

Fig. 1: The one-layer version of the shallow-water model exhibits strong barotropic instability for a basic meridional velocity profile of cosine-square shape (Kuo, 1973). Fields are shown after 10 days of model evolution. Units are $m$ for heights; reference arrow (at top): $54 \mathrm{~ms}^{-1}$ for winds. (After Todling and Ghil, 1991.) 

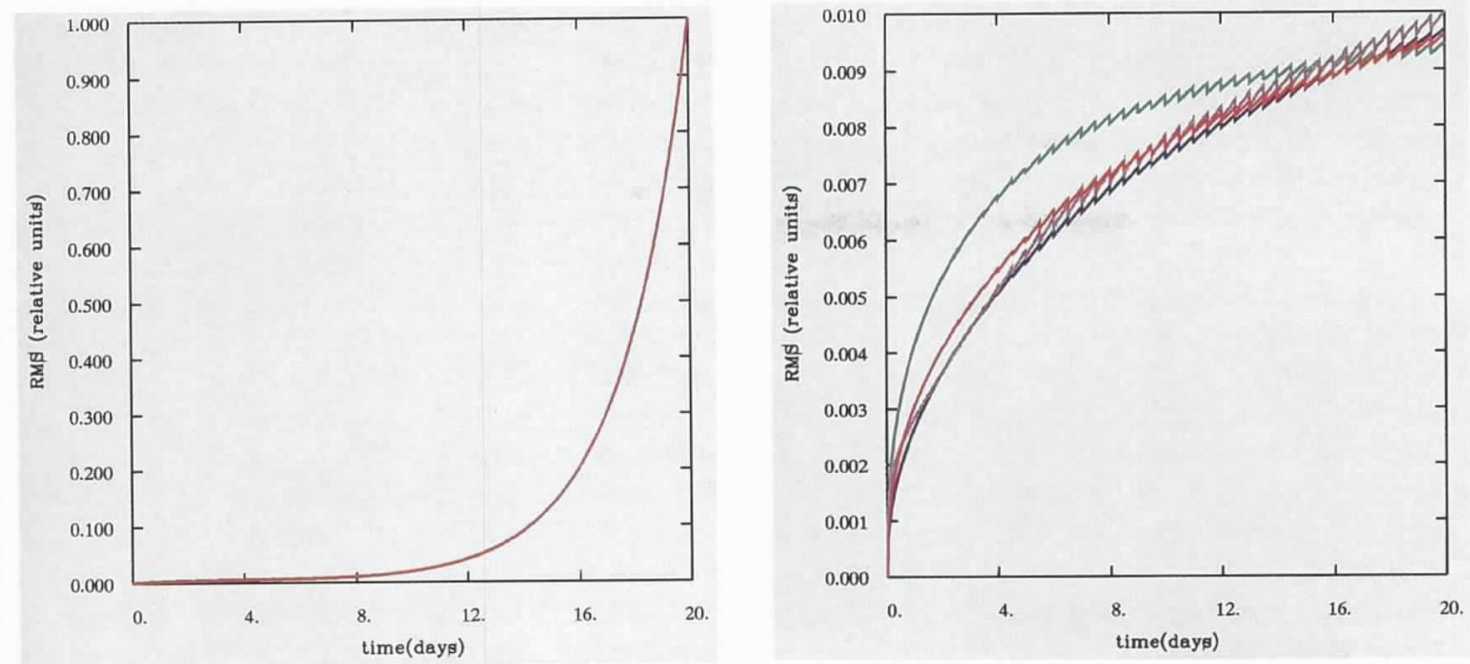

Fig. 2: Expected root-mean-square (RMS) deviation for zonal velocity (blue), meridional velocity (green), heights (purple), and total energy (red) from a Kalman filter applied to an unstable shallow-water model. The panel on the left shows the case without update by observations; curves are normalized by their maximum values and coincide due to the common growth rate of the unstable mode. Panel on the right shows curves for the case with update by observations every 12 hours; curves are normalized by corresponding values in the left panel.

U AT EQUATOR

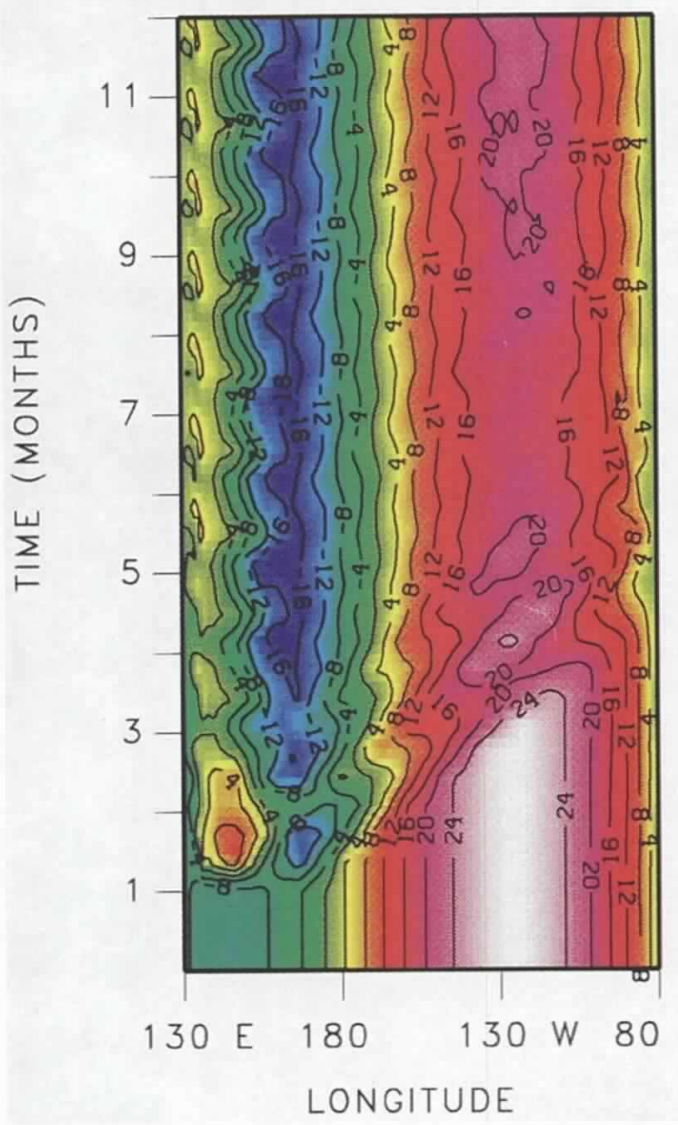

H AT EQUATOR

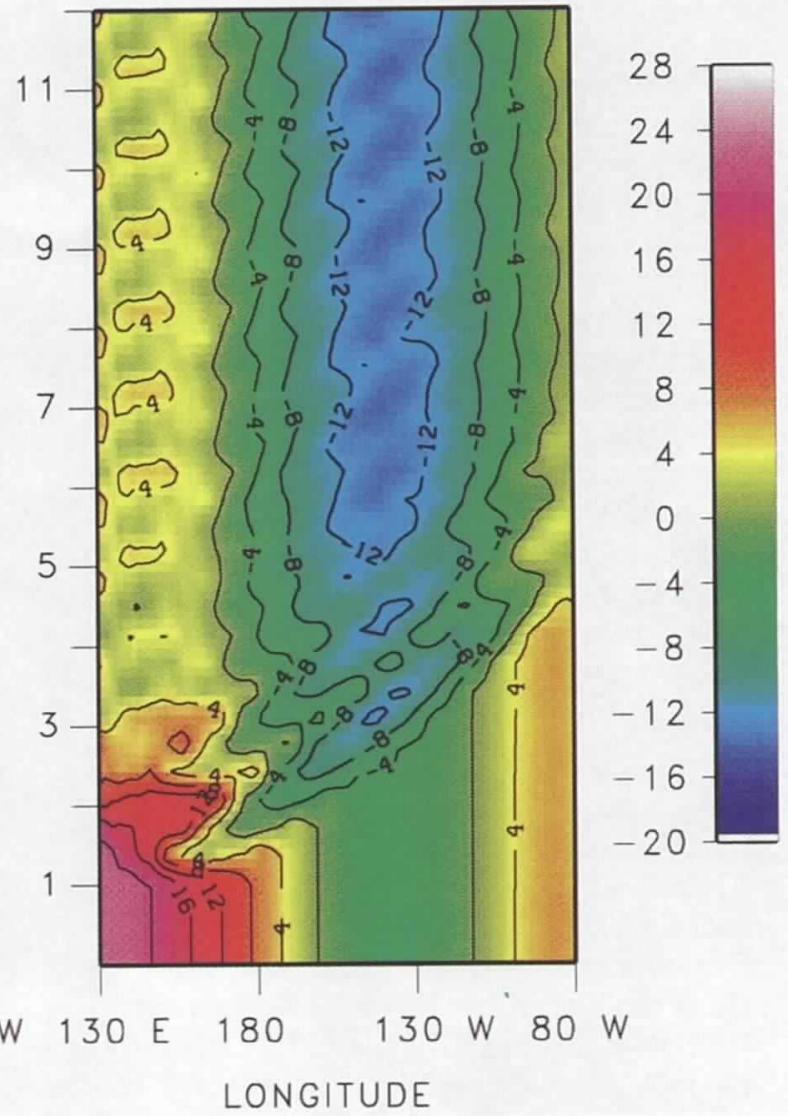

. . . the Kalman filter

results in a substantial

reduction of the initial error, even for a limited

number of

observations . . .
Fig. 3: Data assimilation with an optimal interpolation (OI) scheme in the tropical Pacific. Model anomalies are shown when observations of height and zonal velocity are made only along a meridional line in the western part of the basin, at $140^{\circ} \mathrm{E}$. Zonal-velocity anomaly (left), and thermocline-depth anomaly (right). Values are in nondimensional units, with a contour interval of four units. (After Hao, 1991.) 
of error reduction on location and on the oceanographic field that is being updated arises from the special properties of equatorial waves, which propagate information from data-sparse to datarich regions of the ocean and transfer information from one field variable (height or velocity) to another.

What can be expected from a data-assimilation method in the presence of strong nonlinearities? Miller and Ghil (1990) address this issue by applying three versions of the extended Kalman filter and several variational methods to a number of geophysical models of increasing complexity. Their provisional answer is that the most advanced assimilation methods are expected to capture transitions between different flow regimes and to provide reliable error estimates in cases of chaotic behavior.

\section{Predictability Studies For The Ocean And Atmosphere}

Our predictability studies have focused on two complementary aspects of the problem: instabilities in the open ocean and coastal regions, which limit predictability; and the stability of localized coherent vortex structures, which enhance predictability. We have found shortwave instabilities with rapid growth rates in both a coupled density front with zero potential vorticity (Paldor and Ghil, 1990) and a two-layer coastal front with the interface emerging at some distance from the coast (Paldor and Ghil, 1991). We are proceeding to the nonlinear, viscous treatment of the isolated front, which corresponds to the oceanographically most interesting case of the Gulf Stream north wall.

Concerning the stability of coherent vortices, we have demonstrated that, in agreement with field observations and numerical experiments. stationary (Sakuma and Ghil, 1990) as well as eastward- and westward-traveling (Sakuma and Ghil, 1991) vortex pairs are linearly stable. Our results provide a stronger criterion for the stability of such pairs than was previously available. The physical basis of these results can be explained by the stabilizing effect of rotation on geophysical flows in Sakuma and Ghil (1992).

\section{Modeling Of The Coupled System}

The oceanic global circulation model (OGCM) of the Geophysical Fluid Dynamics Laboratory at Princeton University and the atmospheric global circulation model (AGCM) of UCLA are the components of our coupled GCM. The AGCM provides the wind stress, heat and freshwater fluxes to the OGCM, and the OGCM returns sea-surface temperature (SST) to the AGCM (Mechoso et al., 1991a). The OGCM has a Global version (Bryan and Cox, 1967: Cox and Bryan. 1984) and a Tropical Pacific version with enhanced resolution in the equatorial region (Philander and Pacanowski, 1980). They cover the ocean in the latitude belts from $60^{\circ} \mathrm{S}$ to $60^{\circ} \mathrm{N}$ and from $28^{\circ} \mathrm{S}$ to $50^{\circ} \mathrm{N}$, respectively. The northernmost and southernmost parts of the domains are relaxed towards the observed climatology in both salinity and temperature fields. Incorporation of a sea-ice module is under way.

The AGCM has been developed under the direction of $\mathrm{A}$. Arakawa, with the participation of his colleagues and students (Arakawa and Lamb, 1977). This effort has influenced similar developments around the world during the last 30 years. In the middle 1970s, a version of the model was implemented at the US Naval Environmental Prediction Research Facility and the Fleet $\mathrm{Nu}-$ merical Weather Center, both in Monterey, California. This version evolved into the operational NOGAPS (Navy Operational Global Atmospheric Prediction System) forecasting system (Rosmond 1981). The same version is extensively used for forecasting and climate studies at the Meteorological Research Institute in Tsukuba, Japan (Tokioka et al., 1984).

The current version of the model has been used since the early 1980 s at UCLA and Colorado State University (Randall et al., 1985). The distinctive feature of this latter version is the treatment of the planetary boundary layer (PBL), which is considered well-mixed and is represented by the model's bottom layer, whose variable depth is predicted (Suarez et al., 1983). The PBL parameterization is crucial for modeling heat and momentum fluxes at the ocean-atmosphere interface. Constant effort is dedicated to improvement of the finite-difference schemes and parameterizations of physical processes included in the model and to optimization of its computer code.

The coupled GCM produces a realistic simulation of the seasonal cycle (Mechoso et al., 1991a), without any flux correction. Figures 4 and 5 show the simulated SST field for July, and the time series of simulated SST at the equator, respectively. Figure 4 depicts realistic configurations for the warm pool in the western Pacific and the cold tongue in the eastern Pacific. Figure 5 shows that the east-west temperature gradient is maintained throughout the year by the coupled system, with the extent of cold water largest in July. There is no evidence of significant climate drift. which is a major concern in modeling of the coupled system (Neelin et al., 1992). The successful simulation of the seasonal cycle is a prerequisite for using the coupled GCM in support of our planned data assimilation and predictability studies. We plan to implement, for the OGCM, a four-dimensional data assimilation scheme based on OI and successive corrections.

The computer code of the coupled GCM is being restructured as part of our participation in the Corporation for National Research Initiatives (CNRI) Gigabit Testbed Initiative. In the final stages of this task, computations will be distributed among several supercomputers connected by a 

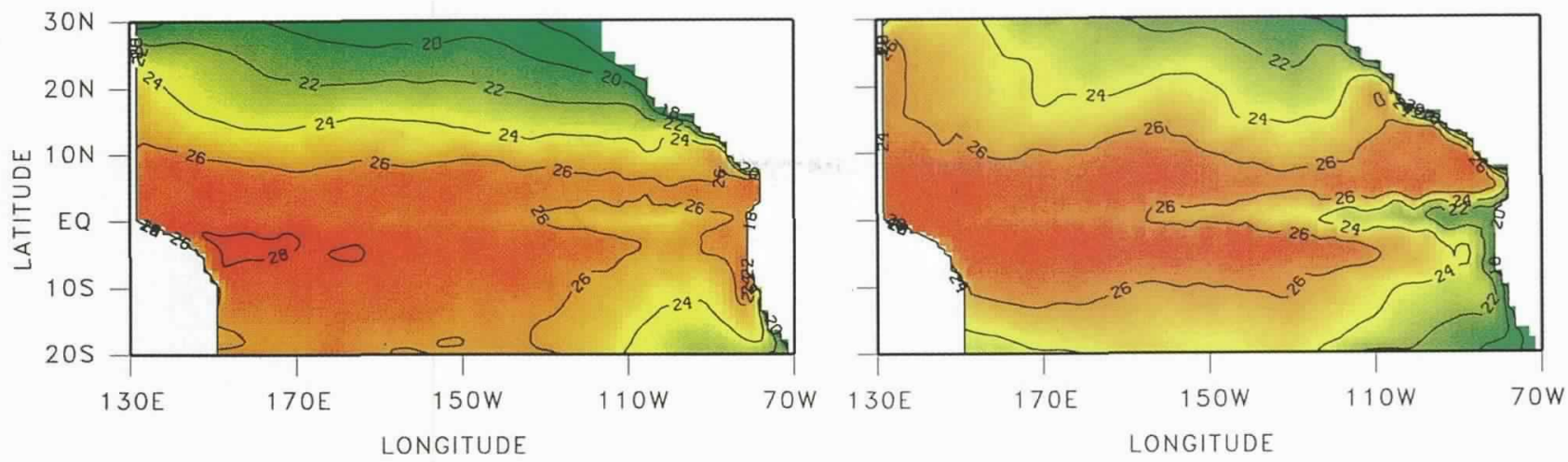

Fig. 4: Sea-surface temperature for January (left) and July (right) corresponding to year 7 in a simulation with the coupled oceanatmosphere GCM. Units are ${ }^{\circ} \mathrm{C}$. (After Mechoso et al., 1991a.)

wide-area high-speed (gigabit per second) network (Mechoso et al., 1991b). Other avenues of parallelization for SIMD (single-instruction/multiple data) and MIMD (multiple-instruction/multiple data) architectures are being tentatively explored on simplified GCMs by Ghil et al. (1991b).

\section{Concluding Remarks}

Methodology for data assimilation and predictability studies on various time scales has been developed and tested on simple models and is ready to be implemented for the UCLA coupled atmosphere-ocean GCM. At the same time, simulations of the seasonal cycle and interannual variability with the coupled GCM are being evaluated. A number of interesting results on data assimilation and predictability for the ocean have been obtained in the process.

In the area of data assimilation, we have evaluated the relative merits of various assimilation methods for the atmosphere and oceans. We have shown the ability of the Kalman filter to track vigorous barotropic and baroclinic instabilities and of the extended Kalman filter to track regime changes in chaotic and stochastically perturbed flows. In the area of predictability, our results include the impact of physical parameterizations (such as that for radiative effects in the AGCM and vertical mixing in the OGCM) on the performance of the coupled system; the existence of shortwave instabilities in frontal structures, both near coasts and in the open ocean; and a theoretical justification of the stability of localized coherent vortex structures in the ocean.

As part of the technological basis for implementing advanced assimilation methods and performing extensive predictability studies on coupled GCMs, we also are exploring modern computer architectures and networks. We expect the coming decade to be one of great excitement for the description, understanding, and prediction of the coupled ocean-atmosphere system.

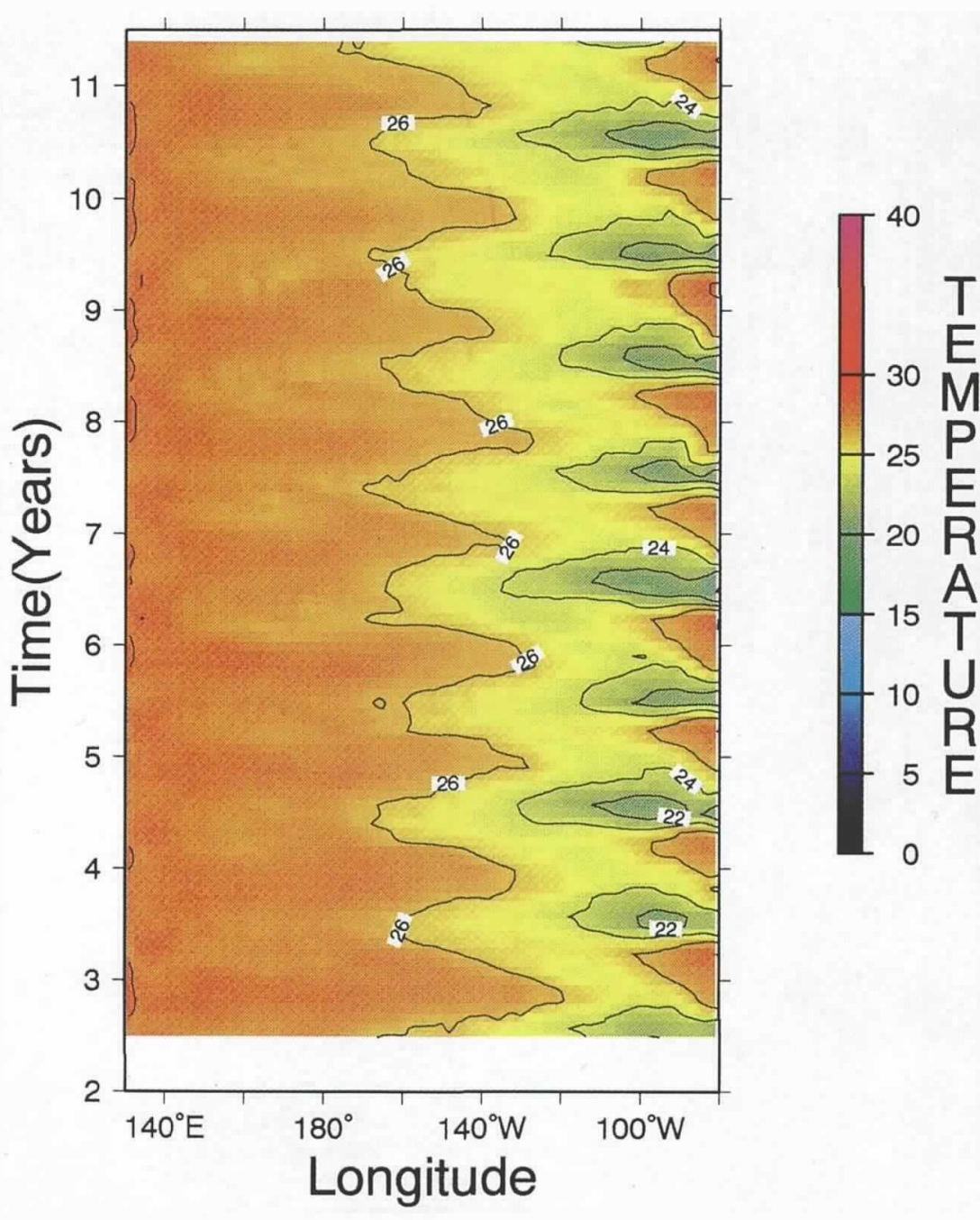

Fig. 5: Time variation of sea-surface temperature at the equator from a simulation with the coupled ocean-atmosphere $G C M$. Units are ${ }^{\circ} \mathrm{C}$. (After Mechoso et al., 1991a.)

\section{Acknowledgements}

It is a pleasure to thank the other participants in this project: Y. Chao, Y. Feliks, M. Fisher, Z. 
Hao, S. Jiang, C.-C. Ma, N. Paldor, H. Sakuma, J.A. Spahr, R. Todling, and W.M. Weibel. Many useful discussions with A. Arakawa, D. Halpern, P. Malanotte-Rizzoli, R.N. Miller, and J.D. Neelin are gratefully acknowledged. The manuscript was typed by $\mathrm{C}$. Wong; Figures 1 and 2 were prepared by R. Todling, Figure 3 by Z. Hao, and Figures 4 and 5 by W.M. Weibel. This research was supported by UCAR/INO under contract S8757 and by ONR under contract N000-14-87K-0331 and grant N00014-89J-1845. Additional support was provided by NSF under grant OCE89-18439, NASA under grant NAG5-713, NASA/ JPL under contract 958658 . NIGEC under contract UCD/WESTGEC 9H12, INCOR, NSF and DARPA under Cooperative Agreement NCR8919038 with CNRI, and by a Guggenheim Fellowship to M. Ghil.

\section{References}

Arakawa, A. and V.R. Lamb, 1977: Computational design of the basic dynamical processes of the UCLA general circulation model. Methods in Computational Physics, 17 , Academic Press, 173-265.

Bengtsson, L., M. Ghil and E. Källén, 1981: Dynamic Meteorology: Data Assimilation Methods, Springer-Verlag. New York, $330 \mathrm{pp}$.

Bryan, K. and M.D. Cox, 1967: A numerical investigation of the oceanic general circulation. Tellus, 19, 54-80.

Cox, M.D. and K. Bryan, 1984: A numerical model of the ventilated thermocline. J. Phys. Oceanog. 14, 674-687.

Daley, R.. 1991: Atmospheric Data Analysis, Cambridge Univ. Press, Cambridge, England. $460 \mathrm{pp}$.

Ghil, M., 1989: Meteorological data assimilation for oceanographers. Part I: Description and theoretical framework. Din. Atmos. Oceans, 13, 171-218.

1990: Sequential estimation in meteorology and oceanography: Theory and numerics. In: Proc. Intl. Symp. Assimilation of Observations in Meteorology and Oceanography, Clermont-Ferrand, World Meteorological Organization, Geneva, pp. 85-90.

, and P. Malanotte-Rizzoli, 1991: Data assimilation in meteorology and oceanography. Adv. Geophys., 33, 141-266.

, S. Cohn, J. Tavantzis, K. Bube, and E. Isaacson, 1981: Applications of estimation theory to numerical weather prediction. Dynamic Meteorology: Data Assimilation Methods, L. Bengtsson, M. Ghil and E. Källén, eds., Springer-Verlag, 139-224.

, M. Kimoto and J.D. Neelin, 1991 a: Nonlinear dynamics and predictability in the atmospheric sciences. Rev. Geophys., Stuplement (U.S. Natl. Report to I.U.G.G. 1987-1990). 46-55.

C.L. Keppenne. G.C. Fox, J.W. Flower, A. Kowala, J.O. Dickey, J.J. Rosati, P.N. Papaccio, J.F. Shepanski, and G. Spadaro, 1991b: Quest, Technology at TRW Space \& Defense Sector. 13, 2, 54-64

Hao, Z., 1991: Data Assimilation and Wind-Stress Error Correction in a Forced Tropical Ocean Model, M.S. thesis. University of California Los Angeles, $57 \mathrm{pp}$.

Kalman, R.E., 1960: A new approach to linear filtering and prediction problems. Trans. ASME, Ser. D, J. Basic Eng., 82, 95-108.

Kuo, H.L., 1973: Quasi-geostrophic flows and instability theory. Adv. Appl. Mech., 13, 247-330.

Mechoso, C.R., C.C. Ma. J.D. Farrara. and J.A. Spahr, 1991a: Simulations of interannual variability with a coupled atmosphere-ocean general circulation model. Preprints Fifth Conf. Climate Variations, Amer. Meteor. Soc., Boston, pp. J1-J4.

,C.-C. Ma, J.D. Farrara, J.A. Spahr, and R.W. Moore. 1991b: Distribution of a coupled atmosphere-ocean general circulation model across high-speed networks. Collection of Technical Papers. Fourth Int. Symp. Comp. Fluid Dynamics.. Davis, California, pp. 795-800.

Miller, R.N., and M. Ghil, 1990: Data assimilation in strongly nonlinear current systems. Proc. Intl. Symp. Asstmilation of Observations in Meteorology and Oceanography, Clermont-Ferrand World Metcorological Organization, Geneva, pp. 93-98.

Neelin, J.D., M. Latif, M.A.F. Allaart. M.A. Cane. U. Cubasch, W.L. Gates, P.R. Gent, M. Ghil, C. Gordon, N.C. Lau, C.R. Mechoso, G.A. Meehl, J.M. Oberhuber. S.G.H. Philander, P.S. Schopf, K.R. Sperber, T. Tokioka, J. Tribbia, and S.E. Zebiak, 1992: Tropical air-sea interaction in general circulation models. Climate Dinamics, 7. 73-104.

Paldor, N., and M. Ghil, 1990: Finite-wavelength instabilities of a coupled density front. $J$. Phys. Oceanogr., 20, 114123.

, and M. Ghil, 1991: Shortwave instabilities of coastal currents. Geophys. Astrophys. Fluid Dyn., 58, 225-241.

Philander, S.G.H. and R.C. Pacanowski, 1980: The generation of equatorial currents. J. Geophls. Res., 85, 1123-1136.

Randall. D.A.. J.A. Abeles. and T.G. Corsetti. 1985: Seasonal simulations of the planetary boundary layer and boundary-layer stratocumulus clouds with a general circulation model. $J$. 4tmos. Sci., 42, 641-676.

Rosmond. T.E., 1981: NOGAPS: Navy Operational Global Atmospheric Prediction System. Preprints Fifih Conference Numerical Weather Prediction. Amer. Meteor. Soc., Boston, pp. 74-79.

Sakuma, H. and M. Ghil, 1990: Stability of stationary barotropic modons by Lyapunov's direct method. J. F/uid Mech., 211, 393-416.

, and M. Ghil, 1991: Stability of propagating modons for small-amplitude perturbations. Phys. Fluids . A, 3 . 408-414.

, and M. Ghil, 1992: Reply to comments by P. Ripa. Phys. Fluids A, 4, 464-466.

Suarez, M.J., A. Arakawa and D.A. Randall, 1983: The parameterization of the planetary boundary layer in the UCLA general circulation model: formulation and results. Mon. Wea. Rev., /1I, 2234-2243.

Todling. R. and M. Ghil. 1990: A Kalman filter for a twolayer two-dimensional shallow-water model. Proc: Intl. Symp. Assimilation of Observations in Meterorology and Oceanography, Clemont-Ferrand, World Meteorological Organization, Geneva, pp. 454-459.

, and M. Ghil, 1991: Tracking atmospheric instabilities with the Kalman filter. Preprints Ninth Conference Numerical Weather Prediction, Amer. Meteor. Soc.. Boston, pp. 177-180.

Tokioka. T., K. Yamazaki, I. Yagai and A. Kitoh, 1984: A description of the Meteorological Research Institute atmospheric general circulation model (the MRI-GCMI), Technical Report of the Meteorslogical Institute. No. 13. $249 \mathrm{pp} . \square$ 